\title{
Which microbial factors really are important in Pseudomonas aeruginosa infections?
}

\author{
Audrey Crousilles', Eve Maunders', Sean Bartlett ${ }^{2}$, Catherine Fan', Emem-Fong Ukor ${ }^{3}$, \\ Yassmin Abdelhamid ${ }^{1}$, Ysobel Baker ${ }^{2}$, Andres Floto ${ }^{3}$, David R Spring ${ }^{2}$ and Martin \\ Welch $^{1^{*}}$
}

\author{
1. Department of Biochemistry (Hopkins Building), Tennis Court Road, \\ Cambridge CB2 1QW, United Kingdom. \\ 2. Department of Chemistry, Lensfield Road, Cambridge CB2 1EW, United \\ Kingdom. \\ 3. Department of Respiratory Medicine, Cambridge Biomedical Campus, \\ Wellcome Trust/MRC Building, Hills Road, Cambridge, CB2 OXY, United \\ Kingdom. \\ *Corresponding author (mw240@cam.ac.uk).
}

Keywords: TnSeq, RNASeq, Pseudomonas aeruginosa, infection, virulence, antimicrobial agents, metabolism, cystic fibrosis.

\begin{abstract}
Over the last two decades, tens of millions of dollars have been invested in understanding virulence in the human pathogen, Pseudomonas aeruginosa. However, the top "hits" obtained in a recent TnSeq analysis aimed at identifying those genes that are conditionally essential for infection did not include most of the known virulence factors identified in these earlier studies. Instead, it seems that $P$. aeruginosa faces metabolic challenges in vivo, and unless it can overcome these, it fails to thrive and is cleared from the host. In this review, we look at the kinds of metabolic pathways that the pathogen seems to find essential, and comment on how this knowledge might be therapeutically exploited.
\end{abstract}

A changing landscape. Pseudomonas aeruginosa is an opportunistic human pathogen - one that has the dubious accolade of featuring regularly among the "top ten" offenders in lists of common hospital "superbugs" worldwide. The organism is capable of causing infections in a wide range of tissue types (especially in immunocompromised hosts) although it exhibits a particular predilection for soft tissues, where the resulting infections can become either acute or chronic. By definition, chronic infections fail to clear through normal immune intervention or following antibiotic treatment, and can often persist for months or even years without resolution. For example, by their 'teens, the airways of many cystic fibrosis (CF) patients often become chronically-colonized by $P$. aeruginosa, and these infections can persist for decades, in spite of regular aggressive antibiotic scourings' [1]. By contrast, acute infections often begin locally, but can rapidly spread to become systemic. Such infections can kill in a matter of days, and for this reason, $P$. aeruginosa remains the scourge of hospital burns units. The key question is, why are the outcomes of these two types of infection - chronic and acute - so radically different? Recent efforts to address this question have forced us to reconsider our previously rather "anthropocentric" notions about which microbial factors really are important for maintaining 
successful infection. It turns out that the answer(s) to this question are not necessarily what we thought. In this review we assess how these recent contributions may lead to a change in our perception of the "virulence paradigm" that has guided research into $P$. aeruginosa pathogenicity for the last few decades.

Some History. First, a bit of background. P. aeruginosa has long been known to be the archetypal "secretor" and its large (ca. 6.4 Mbp) genome encodes an extensive repertoire of secreted virulence factors [2]. Of these, proteases seem to play a particularly important role. Indeed, of the 5568 open reading frames (ORFs) encoded in the genome of the type strain, PAO1, 155 (2.8\%) are listed as proteases in the MEROPS database [3]. Moreover, in the right conditions, the manifold secretory systems (six at the last count) encoded by $P$. aeruginosa churn out vast quantities of these tissuedamaging exoenzymes, which in addition to the proteases, also include phospholipases, exotoxins and endotoxins (Figure 1). A vivid snapshot of the complexity and diversity of the secretome can be garnered by inspection of any of the manifold 2D-PAGE analyses of $P$. aeruginosa exoproteins that have been carried out over the years $[4,5]$. The importance of these virulence factors in infection was inferred from that fact that mutants defective in their synthesis, regulation or secretion often displayed reduced pathogenicity in e.g., rodent pulmonary models of infection. This was especially true of "pleiotropic" mutants affecting multiple virulence phenotypes. For example, the cell-cell signalling mechanism, quorum sensing (QS) has been shown to exert decisive control over the synthesis of many secreted virulence factors in $P$. aeruginosa $[4,6]$. Consistent with this, QS mutants display drastically reduced virulence in vivo $[7,8]$. The inescapable conclusion from these studies was that "virulence factors" are a (if not the) key tactical weapon in the infection strategy of $P$. aeruginosa, and this line of thinking has dominated the direction of research in the area since its inception.

In parallel with the work being carried out on virulence factors, an influential hypothesis began to develop which posited that infection type (acute versus chronic) may be integrally-linked with growth mode. Essentially (so the argument goes) chronic infections are mostly associated with the sessile or "biofilm-like" mode of microbial growth, whereas acute infections involve cells whose physiology more closely resembles that observed in planktonic cultures $[9,10]$ (Figure 2). This was (and remains) an attractive hypothesis for two reasons. First, planktonic cultures of $P$. aeruginosa are known to secrete virulence factors in far greater quantity than their sessile counterparts; a feature that correlated well with the apparently more aggressive nature of acute infections. [Recent work has also shown that biofilms not only secrete fewer exoproteins, but also that the spectrum of proteins secreted is different [4].] Second, due to their polysaccharide coating and altered physiology, biofilms are known to be highly-resistant to antibiotics and also display reduced clearance by the host immune system. These are precisely the features that we associate with chronic infections. Moreover, and consistent with the overall notion that chronic infections are associated with reduced virulence, $P$. aeruginosa isolates harvested from CF patients frequently display impaired virulence factor production (often due to mutation of the master pleiotropic regulator of QS, las $R$ [11]) and/or increased antibiotic resistance e.g., due to the acquisition of mutations in genes encoding repressors of normally-cryptic antibiotic efflux pumps [12].

In the light of the above, and if we had access to technologies that would allow us to "ask the cell" which of its hardware really is important for infection, we would expect to see a list dominated by virulence factors, genes involved in lifestyle decisions, antibiotic resistance, "social functions" (quorum sensing) and so on. Fortunately, such a technology does now exist: TnSeq ([13], Figure 3). This technique exploits the quantitative nature of next-generation DNA sequencing to measure the abundance of a particular transposon ( $\mathrm{Tn}$ ) insertion mutant among a library comprising hundreds of 
thousands of individual mutants disrupted in different genes. The utility of TnSeq is that if a library of mutants is introduced into an infection model, those mutants containing Tn insertions in genes that are conditionally essential for infection should be negatively selected, and this will be reflected by a reduction in the corresponding abundance of DNA sequence reads associated with those gene(s) compared with either the input pool or with DNA harvested from a similarly inoculated in vitro axenic culture. Based on recent TnSeq analyses (see below), it seems that our preconceptions about infection may have been unnecessarily skewed towards the role(s) played by virulence factors, and that other areas of microbial physiology make an equal - if not greater - contribution. Moreover, the same work also put paid to the widely-held assumption that gene expression (transcript levels, measured by RNASeq for example) during infection are a good proxy for the relative importance of the corresponding gene in the infection process (as revealed by TnSeq). It turns out that this is not true.

A different picture emerges. Last year, Turner et al. published a ground-breaking study which combined both TnSeq (i.e., mutant fitness profiling) and RNASeq (transcriptome profiling) to investigate which $P$. aeruginosa genes are conditionally important for acute and chronic infections in mice [14]. In their murine model, they used a subcutaneously infected dorsal burn to mimic an acute infection. This type of infection led to rapid sepsis and $100 \%$ mortality in $48 \mathrm{hr}$. By contrast, a surgicallydelivered dorsal excision was used to mimic a chronic infection. When covered with an adhesive bandage to prevent contractile healing such wounds deposit granulation tissue, are recalcitrant to antibiotic clearance, and can persist for many weeks. As a reference sample for comparison of their in vivo RNASeq and TnSeq data, these workers used planktonic cultures grown to mid-log phase in MOPSbuffered defined medium with succinate as a sole carbon source - a condition in which the physiology of $P$. aeruginosa is well-defined.

RNASeq data. Interestingly, the RNASeq analysis revealed that $14 \%$ and $19 \%$ of ORFs were differentially-transcribed ( $>4$-fold change) in the acute ( $24 \mathrm{hr}$ post-infection) and chronic ( 3 days postinfection) models, respectively. Moreover, there was substantial overlap in the genes that were differentially regulated in each case, suggesting that the cues sensed by the bacteria in both types of infection are similar. Reassuringly, many of the modulated genes - the "usual suspects" - were involved in virulence or protein secretion. Indeed, some of the greatest fold-changes in expression were associated with known virulence factors, although the direction of modulation was not always as expected. For example, transcripts encoding pyochelin (siderophore) synthesis, rhamnolipid synthesis and alkaline protease (all known virulence factors) were strongly up-regulated in both acute and chronic infection models, whereas the $p s /$ biosynthetic genes were strongly down-regulated. The latter was unexpected because Psl polysaccharide is the main matrix component of $P$. aeruginosa biofilms [15], and as noted earlier, biofilms are thought to be associated with the development of chronic infections. However, these were minor incongruities compared with the picture emerging from the parallel TnSeq analyses.

TnSeq data. As in the RNASeq, a sizeable proportion of the mutants displayed differential fitness in the acute and chronic infection models, and some interesting general patterns became evident. For example, the TnSeq data indicated that flagellum-based motility is clearly extremely important in acute but not chronic infection. In contrast, the Type III and Type VI secretion systems were important for fitness in chronic, but not acute infections. This was somewhat unexpected since laboratory experiments have shown that these two secretion systems are reciprocally-regulated in vitro: Type III secretion is thought to be primarily associated with planktonic growth (and therefore, with the development of acute infections) whereas Type VI systems are thought to be primarily associated with biofilm growth (i.e., chronic infection (Figure 2) and $[9,10])$. Presumably, living cells in "real" infections 
are less dogmatic. Alternatively, the distinction between what constitutes planktonic and biofilm-like growth in vivo is more blurred than we think. Another intriguing finding was that the genes encoding the $p s /$ biosynthetic cluster contribute strongly to fitness in both the acute and chronic infection models. Recalling that RNASeq indicated that transcription of the $p s /$ gene cluster was down-regulated in these infection types, this too was unexpected. However, the ps/ example was not unique - indeed, for most classes of gene, RNASeq was generally found to be a very poor predictor of genes important for fitness in the wound models. Critically though, there were some notable exceptions to this trend - primarily involving genes that encode enzymes involved in metabolism - and for the remainder of this review, we will focus on these.

You are what you eat... For some functional categories of gene - especially those involved in primary metabolism - expression levels were found to be a good predictor of mutant fitness. Indeed, it seems that $P$. aeruginosa "rewires" its metabolic pathways during infection to take advantage of the particular set of nutrients available in the host environment. So what kinds of nutrient does $P$. aeruginosa eat as it tucks into a nice meal of mammal? The RNASeq/TnSeq analysis of Turner et al allowed - for the first time - the in vivo dietary preferences of $P$. aeruginosa to be inferred. Projection of the RNASeq data for metabolic genes onto the KEGG pathways map for $P$. aeruginosa revealed some unexpected results. It turns out that $P$. aeruginosa - previously thought to be an amino acid lover [16] (which would be commensurate with the plethora of proteases encoded in its genome) - is particularly partial to long-chain fatty acids. The faoAB genes (encoding the 2-enoyl-CoA hydratase / 3-hydroxyacyl-CoA dehydrogenase subunit (FaoA) and 3-oxoacyl-CoA thiolase subunit (FaoB) of the fatty acid oxidase complex [17]) were up-regulated in the acute infection model, and the same genes displayed fitness defects when disrupted by Tn insertions. Moreover, a defined faoA deletion mutant was attenuated in both chronic and acute infection models, suggesting that fatty acid oxidation plays a key role in survival in vivo.

$2 \mathrm{C}$ or not $\mathbf{2 C}$ ? The problem with utilizing fatty acids is that they are broken down via $\beta$-oxidation to acetyl-CoA units. These acetate moieties are then combined with oxaloacetate to yield citrate, which then enters the TCA cycle. Normally, as they progress around the cycle, these two acetate-derived C atoms are liberated as $\mathrm{CO}_{2}$. This means that the two input $\mathrm{C}$ atoms (acetate moieties) cannot be used to generate biomass. The cell can therefore generate energy (i.e., NADH and thence ATP equivalents) but cannot grow; gluconeogenesis is not possible when fatty acids are the sole $\mathrm{C}$ source. However, bacteria have evolved a clever solution to this problem. Prior to the $\mathrm{CO}_{2}$-producing steps of the TCA cycle, a proportion of the carbon skeletons are "siphoned off" the main sequence of TCA cycle reactions in a pathway known as the glyoxylate shunt (Figure 4). Here, isocitrate is cleaved by isocitrate lyase (encoded by aceA) to yield glyoxylate and succinate (which re-enters the TCA cycle following the oxidative decarboxylation steps). The glyoxylate is not wasted though; malate synthase $\mathrm{G}$ (encoded by $g / c B$ in $P$. aeruginosa) combines glyoxylate with an additional acetyl-CoA molecule to yield the gluconeogenic precursor, malate. The net effect of this is that $\mathrm{C}$ skeletons are preserved for anabolic reactions and the creation of biomass $[18,19]$. Consistent with this proposed redirection of carbon flux, Turner et al found that the transcripts encoding enzymes involved in the oxidative decarboxylation steps of the TCA cycle (isocitrate dehydrogenase(s) and $\alpha$-ketoglutarate dehydrogenase) are down-regulated, whereas the transcript encoding the first enzyme of the glyoxylate shunt (aceA) was up-regulated [14].

The importance of the glyoxylate shunt in infections has been noted before. Lindsey et al. found that an isocitrate lyase (aceA) mutant displays reduced virulence in a rat lung infection model and noted that isocitrate lyase activity is often up-regulated in CF-derived isolates adapted to chronic infection 
[20]. More recently, Fahnoe et al inactivated both aceA and $g / c B$ in $P$. aeruginosa i.e., completely inactivating the glyoxylate shunt [21]. This double mutant was completely cleared by $48 \mathrm{hr}$ postinfection in a mouse pulmonary infection model, indicating that the shunt is absolutely essential for colonization in vivo. Remarkably, the same team also identified a collection of small molecules that could simultaneously inhibit both isocitrate lyase and malate synthase $\mathrm{G}$ in laboratory cultures of $P$. aeruginosa. Unfortunately, Fahnoe et al. did not report on whether their "top hit" compounds could resolve in vivo infections - presumably, these compounds may be toxic in mammalian systems, but the overall approach still looks promising. Indeed, nature has already exploited this strategy; recent work has shown that the human enzyme Irg1 synthesizes large quantities of itaconate (an inhibitor of isocitrate lyase) during macrophage activation, directly contributing towards the antimicrobial activity of these cells (reviewed in [22]). However, and in a remarkable evolutionary tit-for-tat, P. aeruginosa (along with other pathogens such as Yersinia pestis) has also acquired the ability to degrade itaconate, and can even live off this compound as a sole $C$ source ([23]). Furthermore, recent data suggest that $P$. aeruginosa can also degrade other isocitrate lyase inhibitors, such as nitropropionic acid (produced by some plants and fungi) via the nitronate monooxygenase activity of the gene encoded by PA4202 $(n m o A)[24]$.

Interestingly, recent ${ }^{13} \mathrm{C}$ flux analyses have indicated that unlike the situation in Escherichia coli, where ace $A$ is only expressed during growth on acetate (or acetate-producing substrates), in P. aeruginosa, there is significant flux through the pathway even during growth on glucose [25]. This suggests that the shunt may play a more general role in the physiology of the organism beyond simply redirecting $C$ skeletons for gluconeogenesis. Indeed, inhibition of the glyoxylate shunt may deliver a double whammy to $P$. aeruginosa; our own team recently showed that isocitrate lyase activity stimulates Type III Secretion during microaerobic growth, suggesting that the absence of the glyoxylate shunt not only results in metabolic insufficiency - it also diminishes virulence per se [26]. Moreover, itaconate could suppress Type III Secretion when added to microaerobic cultures in vitro. Crucially in this regard, the RNASeq/TnSeq data of Turner et al. strongly suggest that oxygen limitation plays a key role in infection - the anaerobic regulator [27] encoded by anr was among the most strongly attenuated Tn mutants that they identified, especially during acute infection [14]. These data suggest that oxygen limitation may be a common feature of many $P$. aeruginosa infections.

Quite why $P$. aeruginosa chooses a metabolic strategy that is dependent upon the glyoxylate shunt (instead of utilizing more "available" nutrients) is not clear. However, inspection of the rank-ordered list of attenuated Tn mutants obtained by Turner et al reveals that insertions in $g / p D$ (glycerol 3phosphate dehydrogenase) were also essentially avirulent in vivo. GlpD converts glycerol 3-phosphate into dihydroxyacetone phosphate, and a mutant in $g / p D$ was recently shown to produce lower levels of certain virulence determinants (pyocyanin and pyoverdine) in vitro [28]. One major possible source of glycerol in vivo is phospholipids, which are broken down to yield fatty acids and glycerol.

To get a better insight into what kinds of nutrient are on the $P$. aeruginosa menu in a mouse, Turner et al. compared the biosynthetic pathways expressed during growth on minimal succinate media (where most biosynthetic pathways are necessarily "on") with those expressed in vivo. The logic here was that biosynthetic pathways are generally only transcribed when the corresponding pathway product is not available from the environment (after all, why waste valuable metabolic resources synthesizing say, an amino acid, if the same amino acid is plentiful in the growth media?). Therefore, by comparing these two datasets, they could infer which nutrients might be available to $P$. aeruginos in vivo during wound infections. The results were surprising; non-available metabolites included certain amino acids (glutamate, aspartate, tyrosine, phenylalanine and asparagine) as well as purines, 
$p$-aminobenzoate and B-group vitamins (the precursors for many cofactors). Indeed, and further confirming these results, in vitro-constructed $p a b C$ and purA mutants (deficient in $p$-aminobenzoate and purine biosynthesis, respectively) were avirulent in vivo. In contrast, mutants defective in metabolites predicted to be "available" in vivo were unaffected in virulence. This striking result strongly suggests that components of the pathways required for synthesis of "non-available" metabolites may make excellent targets for the development of new anti-pseudomonal agents.

The situation is slightly different in CF infections. More recently, Turner et al. have been using Monte Carlo simulations to rigorously interrogate a new set of TnSeq data in which the growth requirements of $P$. aeruginosa in CF sputum have been investigated. These data have enabled them to refine the composition of artificial CF sputum medium [29]. The gene fitness requirements in this new medium now so closely resemble that in "real" CF sputum that they are all but indistinguishable, thereby providing a far better laboratory model for the study of CF-associated $P$. aeruginosa infections than previously possible. In the same study, the authors also compared the list of genes that are conditionally essential for the survival of two strains of $P$. aeruginosa (PAO1 and PA14) when grown in minimal medium containing authentic CF sputum as a sole nutrient source. Interestingly, most of the conditionally essential genes were located in the conserved "core" genome of both strains. However, clear differences were observed in the relative essentiality of different genes in each strain. Moreover, and although common pathways (e.g., those for chorismate, panthothenate, diaminopimelate, purines, pyridoxal phosphate, riboflavin and certain polyamines) were deemed essential for infection in both wound [14] and CF infections [29], some important differences were apparent. For example, as outlined earlier, fatty acid oxidation is essential in wound infections but seems to play a much less important role during growth in CF sputum. In general, the authors concluded that CF sputum presents a far less harsh environment compared with that encountered in wound infections, although cynically, this may simply be a reflection of the fact that the in vitro CF model used by those workers contains no immune cells, whereas presumably, an exuberant immune response is mounted in the in vivo wound models. Nevertheless, the results of Turner et al [14, 29] and Fahnoe et al [21] strongly suggest that the targeting specific domains of metabolism may offer a real opportunity to develop some truly innovative antimicrobial interventions. In this regard, it is ironic to note that one of the first antibiotics, sulfanilimide (introduced in the 1930s), targets $p$ aminobenzoate metabolism.

Real-life infections are more complex. One fly-in-the-ointment in the case for targeting specific metabolic nodes is that $P$. aeruginosa isolates which are auxotrophic for certain amino acids arise frequently in chronic CF infections [30]. Indeed, we too have identified auxotrophs in CF sputum that are defective in the synthesis of one or more of the 20 common amino acids, as well as in the synthesis of certain cofactors (MW/E-FU/AF, unpublished data). This is surprising given that the availability of several amino acids (e.g., Gln, Tyr, Asn, Asp) is predicted by Turner et al. to be limiting in CF sputum [29]. However, and as noted by Barth \& Pitt, CF sputum is certainly rich enough in amino acids to support the growth of some auxotrophs [31]. Additionally, there is even evidence to suggest that high total amino acid concentrations in sputum coincide with acute pulmonary exacerbation episodes and an increased prevalence of auxotrophs [32]. One possibility is that these sputum amino acids are hostderived. If this is so, then the case for targeting $P$. aeruginosa amino acid biosynthesis for possible therapeutic intervention is weakened. Alternatively, the sputum amino acids could be microbiallyderived. In this regard, biofilm and anaerobic planktonic cultures of $P$. aeruginosa (PAO1) grown in defined medium in vitro are known to accumulate large quantities of amino acids [33], presumably due to overflow metabolism. [We note here that the sputum samples used in the study of Tuner et al. that were found to be limiting for Gln/Tyr/Asn/Asp were (understandably) obtained from a patient 
with a low bacterial load [29].] This raises the possibility that the population structure of $P$. aeruginosa in any given chronically-infected CF patient may be self-stabilized via intra-species cross-feeding. For example, Tyr $P$. aeruginosa auxotrophs could be sustained off the tyrosine provided by $\mathrm{Tyr}^{+}$ prototrophs, and so on. If this is indeed the case, then amino acids (and presumably, other metabolites too) can be viewed as bacterially-produced "public goods" in much the same way that virulence factors have been $[34,35]$. While this possibility has not yet been rigorously tested experimentally, there is good evidence to suggest that the population of $P$. aeruginosa in a given sputum sample is both phenotypically and genomically heterogeneous [36-39]. If true and the $P$. aeruginosa population structure is stabilized by cross-feeding of nutrients, then by targeting the synthesis of one or more of these nutrients, the network of interactions that maintains population cohesion should collapse (or at least undergo radical remodelling). However, a potential counter-argument lies in the fact that CF infections are rarely associated with $P$. aeruginosa monocultures; a veritable zoo of microbes can be identified (usually from their 16S rDNA signatures) in many CF sputa [40, 41], raising further opportunities for cross-feeding. Consequently, drugs targeting specific domains of metabolism may need to have rather broad specificity, allowing them to target enzymes in multiple genera of bacteria.

Parting comments. $P$. aeruginosa is not a professional pathogen; in spite of its genomic arsenal of putative virulence determinants, it is primarily an opportunist that has to adapt to the host environment in order to survive. The TnSeq analyses of Turner et al have yielded insights into what the cell itself finds important during this process. The precise physiological adaptations that appear to be truly important are tuned to some extent by the subtle nuances attending the different types of infection in different tissues. However, there is enough common ground between the requirements in these different infection types to raise the hope that generic new anti-pseudomonal compounds might be discovered through this avenue of investigation. This notwithstanding, and aside from the issues raised earlier in this discussion, there are caveats. One is the issue of redundancy. For example, although FaoA/FaoB clearly play an important role in wound infections, the acyl-CoA dehydrogenases that necessarily precede the action of these enzymes were not identified as being conditionally essential in infection. Presumably, this is because multiple copies of genes encoding acyl-CoA dehydrogenases are present in the $P$. aeruginosa genome (seven genes are annotated as putative acylCoA dehydrogenases in PAO1) and inactivation of any one of these genes may be compensated by the residual activity of the other isozymes present. A similar argument could be made for ppGpp generating enzymes. The stress alarmone, ppGpp, is required for amino acid biosynthetic pathway expression [42] and has been implicated in virulence in many bacterial pathogens (reviewed in [43]). However, for a noticeable virulence phenotype to be manifest, both ppGpp-generating enzymes (RelA and SpoT) need to be inactivated [44]. Similarly, cyclic di-GMP is also known to play a key role in determining lifestyle and virulence in $P$. aeruginosa, yet this small molecule is made and sensed by a multitude of gene products [45]. Another potential issue relates to the nature of the medium employed for growth of the TnSeq reference samples (recall that Turner et al used MOPS-buffered succinate medium). This is because the "essential gene set" varies somewhat during growth in vitro in different media (Lee et al, [46]). Although there is an apparent "core" set of ca. 350 genes (many of which are involved in central carbon metabolism) that seem to be essential in most common laboratory growth media, Lee et al identified ca. 200 genes that appear to be conditionally essential in different growth media. Thus, the conclusions of Turner et al will be inevitably biased somewhat by their specific choice of MOPS-succinate for growth of the reference sample. However, and commensurate with the results discussed earlier, Lee et al also found that CF sputum is likely to be rich in amino acids and unsaturated fatty acids [46]. 
Future Perspective. These arguments aside, the results of Turner et al. must at the very least cause us to re-assess our notions about $P$. aeruginosa virulence. The past two decades of $P$. aeruginosa virulence research have been dominated by the role(s) played by quorum sensing and biofilm formation. However, it turns out that although the "key regulators" associated with these phenotypes (retS, IadS, lasR, gacA, gacS, rsmA, rsmY, rsmZ etc - $[9,10,47]$ ) do feature on the "TnSeq attenuatedlist", for the most part they do not make the "top 200" for any infection type. That is not to say that they do not play an important role in infection under some circumstances - they do, and we know that. It's just that some rather more mundane (and perhaps, overlooked?) phenotypes seem to be more important. This may actually be good news; unlike many of the pleiotropic virulence regulators that have become a favoured target for antimicrobial research over the last two decades, the enzymes of central metabolism are generally (i) highly conserved across the bacterial genera, and (ii) contain discrete "drug-able" binding pockets that are amenable to drug development. Moreover, we have historical proof-of-principle that the targeting of metabolic nodes (re: the sulphonamides) works. Given the dearth of new antimicrobials in the pipeline, coupled with the inexorable spread of resistance to existing antibiotics, it seems likely that over the next few years we will see a renewed interest in targeting central metabolism.

\section{Executive Summary.}

- Pseudomonas aeruginosa is an opportunistic pathogen which produces a wealth of secreted virulence factors and secondary metabolites. These secreted factors have been assumed to play a pivotal role in infection.

- New technologies are allowing us to "ask the bacterial cell" which of its genes are important for infection in mammalian systems. For example, RNASeq allows quantitation of transcript levels whereas TnSeq reports on the contribution of specific genes towards fitness in vivo.

- In a recent analysis, RNASeq and TnSeq were used in parallel to investigate which $P$. aeruginosa genes are required for optimal virulence in a murine infection model. Surprisingly, for most classes of gene (including known virulence factors) this analysis revealed that there was very little correlation between transcript abundance and the contribution of the corresponding gene towards overall fitness. However, for certain "metabolic genes", these factors were strongly correlated.

- During some types of infection, $P$. aeruginosa exhibits a predilection for metabolizing fatty acids. Mutants in the fatty acid oxidase complex are severely attenuated in virulence. In addition, the glyoxylate shunt (an anaplerotic pathway which bypasses the oxidative decarboxylation steps of the TCA cycle) makes an important contribution towards fitness in vivo.

- Certain metabolites (amino acids and vitamins) are not available to $P$. aeruginosa during in vivo infections, making the biosynthetic pathways producing these compounds excellent potential targets for the development of novel antimicrobials.

- Not all types of infection elicit the same metabolic requirements in $P$. aeruginosa. Moreover, the relative essentiality of different genes varies between different $P$. aeruginosa strains. 
354

\section{References.}

\section{*Of interest}

\section{** Of considerable interest.}

1. Foweraker J. Recent advances in the microbiology of respiratory tract infection in cystic fibrosis. Br Med Bull. 89:93-110 (2009).

2. Stover CK, Pham XQ, Erwin AL et al. Complete genome sequence of Pseudomonas aeruginosa PAO1, an opportunistic pathogen. Nature. 406:959-64 (2000).

3. Hoge R, Pelzer A, Rosenau F and Wilhelm S. Weapons of a pathogen: Proteases and their role in virulence of Pseudomonas aeruginosa. In: Current Research: Technology and education topics in applied microbiology and microbial biotechnology. Ed: A Mendez-Vilas.

4. Passmore IJ, Nishikawa K, Lilley KS et al. Mep72, a metzincin protease that is preferentially secreted by biofilms of Pseudomonas aeruginosa. J Bacteriol. 197:762-73 (2015).

5. Nouwens AS, Beatson SA, Whitchurch CB et al. Proteome analysis of extracellular proteins regulated by the las and rhl quorum sensing systems in Pseudomonas aeruginosa PAO1. Microbiology. 149:1311-22 (2003).

6. Pearson JP, Pesci EC and Iglewski BH. Roles of Pseudomonas aeruginosa las and rhl quorumsensing systems in control of elastase and rhamnolipid biosynthesis genes. J Bacteriol. 179:5756-67 (1997).

7. Pearson JP, Feldman M, Iglewski BH and Prince A. Pseudomonas aeruginosa cell-to-cell signaling is required for virulence in a model of acute pulmonary infection. Infect Immun. 68:4331-4 (2000).

8. Rumbaugh KP, Griswold JA, Iglewski BH and Hamood AN. Contribution of quorum sensing to the virulence of Pseudomonas aeruginosa in burn wound infections. Infect Immun. 67:585462 (1999).

9. Goodman AL, Kulasekara B, Rietsch A et al. A signaling network reciprocally regulates genes associated with acute infection and chronic persistence in Pseudomonas aeruginosa. Dev Cell. 7:745-54 (2004).

10. Ventre I, Goodman AL, Vallet-Gely I et al. Multiple sensors control reciprocal expression of Pseudomonas aeruginosa regulatory RNA and virulence genes. Proc Natl Acad Sci U S A. 103:171-6 (2006).

11. D'Argenio DA, Wu M, Hoffman LR, Kulasekara HD et al. Growth phenotypes of Pseudomonas aeruginosa lasR mutants adapted to the airways of cystic fibrosis patients. Mol Microbiol. 64:512-33 (2007).

12. Smith EE, Buckley DG, Wu Z, Saenphimmachak $C$ et al. Genetic adaptation by Pseudomonas aeruginosa to the airways of cystic fibrosis patients. Proc Natl Acad Sci U S A. 103:8487-92 (2006).

13. van Opijnen $T$, Bodi $K L$ and Camilli A. Tn-seq: high-throughput parallel sequencing for fitness and genetic interaction studies in microorganisms. Nat. Methods 6:767-772 (2009).

14. Turner KH, Everett J, Trivedi U et al. Requirements for Pseudomonas aeruginosa acute burn and chronic surgical wound infection. PLoS Genet. 10(7):e1004518. doi: 10.1371/journal.pgen.1004518 (2014).

**Turner et al use a combination of TnSeq and RNASeq to assess which genes/transcripts are conditionally important for infection in vivo. They use these data to gauge the types of metabolite that are likely available to $P$. aeruginosa during wound infections. 
15. Jackson KD, Starkey M, Kremer $\mathrm{S}$ et al. Identification of psl, a locus encoding a potential exopolysaccharide that is essential for Pseudomonas aeruginosa PAO1 biofilm formation. J Bacteriol. 186:4466-75 (2004).

16. Palmer KL, Aye LM, Whiteley M. Nutritional cues control Pseudomonas aeruginosa multicellular behavior in cystic fibrosis sputum. J Bacteriol. 189:8079-87 (2007).

17. Sato $S$, Hayashi $M$, Imamura $S$ et al. Primary structures of the genes, $f a o A$ and $f a o B$, from Pseudomonas fragi B-0771 which encode the two subunits of the HDT multienzyme complex involved in fatty acid beta-oxidation. J Biochem. 111:8-15 (1992).

18. Cozzone AJ. Regulation of acetate metabolism by protein phosphorylation in enteric bacteria. Annu Rev Microbiol. 52:127-64 (1998).

19. M. de la Pena Mattozzi, Y. Kang and J. D. Keasling Feast: Choking on acetyl-CoA, the glyoxylate shunt, and acetyl-CoA-driven metabolism. In: Handbook of Hydrocarbon and Lipid Microbiology, K. N. Timmis (ed.), DOI 10.1007/978-3-540-77587-4_116, Springer-Verlag Berlin Heidelberg (2010).

20. Lindsey TL, Hagins JM, Sokol PA, Silo-Suh LA. Virulence determinants from a cystic fibrosis isolate of Pseudomonas aeruginosa include isocitrate lyase. Microbiology. 154:1616-27 (2008).

21. Fahnoe KC, Flanagan ME, Gibson $\mathrm{G}$ et al. Non-traditional antibacterial screening approaches for the identification of novel inhibitors of the glyoxylate shunt in gram-negative pathogens. PLoS One. 7(12):e51732. doi: 10.1371/journal.pone.0051732 (2012).

*A short report showing that the glyoxylate shunt is essential for survival of $P$. aeruginosa in a mouse pulmonary model. The authors also show that certain small molecules can simultaneously target both enzymes of the glyoxylate shunt.

22. Ménage S, Attrée I. Metabolism: pathogens love the poison. Nat Chem Biol. 10:326-7. (2014).

23. Sasikaran J, Ziemski M, Zadora, PK et al. Bacterial itaconate degradation promotes pathogenicity. Nat. Chem. Biol. 10:371-377 (2014).

24. Vercammen K, Wei Q, Charlier D et al. Pseudomonas aeruginosa LysR PA4203 regulator NmoR acts as a repressor of the PA4202 nmoA gene, encoding a nitronate monooxygenase. J Bacteriol. 197:1026-39 (2015).

25. Berger A, Dohnt K, Tielen P, Jahn D, Becker J, Wittmann C. Robustness and plasticity of metabolic pathway flux among uropathogenic isolates of Pseudomonas aeruginosa. PLoS One. 9(4):e88368. doi: 10.1371/journal.pone.0088368. (2014).

26. Chung JC, Rzhepishevska O, Ramstedt M, Welch M. Type III secretion system expression in oxygen-limited Pseudomonas aeruginosa cultures is stimulated by isocitrate lyase activity. Open Biol. 3:120131. doi: 10.1098/rsob.120131 (2013).

*Flux through the glyoxylate shunt not only affects metabolism: it also affects the main virulence determinant of $P$. aeruginosa - the Type III Secretion System.

27. Trunk K, Benkert B, Quäck $N$ et al. Anaerobic adaptation in Pseudomonas aeruginosa: definition of the Anr and Dnr regulons. Environ Microbiol. 12:1719-33. doi: 10.1111/j.14622920.2010.02252.x. (2010).

28. Daniels JB, Scoffield J, Woolnough JL, Silo-Suh L. Impact of glycerol-3-phosphate dehydrogenase on virulence factor production by Pseudomonas aeruginosa. Can J Microbiol. 60:857-63 (2014).

29. Turner KH, Wessel AK, Palmer GC et al. Essential genome of Pseudomonas aeruginosa in cystic fibrosis sputum. Proc Natl Acad Sci U S A. 112:4110-5 (2015). 
**Here, the authors use a combined TnSeq/Monte Carlo approach to assess which $P$. aeruginosa genes are likely to be essential for growth in the CF lung. They build on these findings by formulating an artificial CF sputum medium that is all but indistinguishable from "real" CF sputum.

30. Barth AL, Pitt TL. Auxotrophic variants of Pseudomonas aeruginosa are selected from prototrophic wild-type strains in respiratory infections in patients with cystic fibrosis. J Clin Microbiol. 33:37-40 (1995).

31. Barth AL, Pitt TL. The high amino-acid content of sputum from cystic fibrosis patients promotes growth of auxotrophic Pseudomonas aeruginosa. J Med Microbiol. 45:110-9 (1996).

32. Thomas SR, Ray A, Hodson ME, Pitt TL. Increased sputum amino acid concentrations and auxotrophy of Pseudomonas aeruginosa in severe cystic fibrosis lung disease. Thorax. 55:7957 (2000).

33. Patell S, Gu M, Davenport P, Givskov M, Waite RD, Welch M. Comparative microarray analysis reveals that the core biofilm-associated transcriptome of Pseudomonas aeruginosa comprises relatively few genes. Environ Microbiol Rep. 2:440-8 (2010).

34. Rainey PB, Rainey K. Evolution of cooperation and conflict in experimental bacterial populations. Nature. 425:72-4 (2003).

35. Diggle SP, Griffin AS, Campbell GS and West SA. Cooperation and conflict in quorum-sensing bacterial populations. Nature. 450:411-4 (2007).

36. Chung JC, Becq J, Fraser L, Schulz-Trieglaff O, Bond NJ, Foweraker J, Bruce KD, Smith GP, Welch M. Genomic variation among contemporary Pseudomonas aeruginosa isolates from chronically infected cystic fibrosis patients. J Bacteriol. 194:4857-66 (2012).

37. Williams D, Evans B, Haldenby S et al. Divergent, coexisting Pseudomonas aeruginosa lineages in chronic cystic fibrosis lung infections. Am J Respir Crit Care Med. 191:775-85 (2015).

38. Mowat $\mathrm{E}$, Paterson $\mathrm{S}$, Fothergill $\mathrm{JL}$ et al. Pseudomonas aeruginosa population diversity and turnover in cystic fibrosis chronic infections. Am J Respir Crit Care Med. 183:1674-9 (2011).

*Perhaps the best demonstration yet that the $P$. aeruginosa population in the CF airways is likely to be dynamic and diverse.

39. Foweraker JE, Laughton CR, Brown DF, Bilton D. Phenotypic variability of Pseudomonas aeruginosa in sputa from patients with acute infective exacerbation of cystic fibrosis and its impact on the validity of antimicrobial susceptibility testing. J Antimicrob Chemother. 55:9217 (2005).

40. Twomey KB, Alston M, An SQ et al. Microbiota and metabolite profiling reveal specific alterations in bacterial community structure and environment in the cystic fibrosis airway during exacerbation. PLoS One. 8:e82432. doi: 10.1371/journal.pone.0082432 (2013).

41. Lynch SV, Bruce KD. The cystic fibrosis airway microbiome. Cold Spring Harb Perspect Med. 3:a009738. doi: 10.1101/cshperspect.a009738 (2013).

42. Traxler, MF, Summers SM, Nguyen HT et al. The global, ppGpp-mediated stringent response to amino acid starvation in Escherichia coli. Mol Microbiol. 68:1128-48 (2008).

43. Dalebroux ZD, Svensson SL, Gaynor EC, Swanson MS. ppGpp conjures bacterial virulence. Microbiol Mol Biol Rev. 74:171-99 (2010).

44. Bowden SD, Eyres A, Chung JC et al. Virulence in Pectobacterium atrosepticum is regulated by a coincidence circuit involving quorum sensing and the stress alarmone, (p)ppGpp. Mol Microbiol. 90:457-71 (2013). 
45. Moscoso JA, Mikkelsen $\mathrm{H}$, Heeb $\mathrm{S}$ et al. The Pseudomonas aeruginosa sensor RetS switches type III and type VI secretion via c-di-GMP signalling. Environ Microbiol. 13:3128-38 (2011).

46. Lee SA, Gallagher LA, Thongdee M et al. General and condition-specific essential functions of Pseudomonas aeruginosa. Proc Natl Acad Sci U S A. 112:5189-94 (2015).

${ }^{* *}$ A great description of the TnSeq principle. Here, TnSeq is used to assess the $P$. aeruginosa essential gene set in different growth media in vitro.

47. Brencic A, McFarland KA, McManus HR et al. The GacS/GacA signal transduction system of Pseudomonas aeruginosa acts exclusively through its control over the transcription of the RsmY and RsmZ regulatory small RNAs. Mol Microbiol. 73:434-45 (2009).

48. Filloux A, Michel G, Bally M. GSP-dependent protein secretion in gram-negative bacteria: the Xcp system of Pseudomonas aeruginosa. FEMS Microbiol Rev. 22:177-98 (1998).

49. Ball G, Durand E, Lazdunski A and Filloux A. A novel type II secretion system in Pseudomonas aeruginosa. Mol Microbiol. 43:475-85 (2002).

50. Bleves S, Viarre V, Salacha R, Michel GP, Filloux A, Voulhoux R. Protein secretion systems in Pseudomonas aeruginosa: A wealth of pathogenic weapons. Int J Med Microbiol. 300:534-43 (2010).

51. Filloux A. Protein secretion systems in Pseudomonas aeruginosa: An essay on diversity, evolution, and function. Front Microbiol. 2:155. doi: 10.3389/fmicb.2011.00155 (2011).

52. Franklin MJ, Nivens DE, Weadge JT and Howell PL. Biosynthesis of the Pseudomonas aeruginosa Extracellular Polysaccharides, Alginate, Pel, and Psl. Front Microbiol. 2:167. doi: 10.3389/fmicb.2011.00167 (2011).

53. LaPorte \& Koshland. Phosphorylation of isocitrate dehydrogenase as a demonstration of enhanced sensitivity in covalent modification. Nature 305:286-90 (1983).

\section{Acknowledgements}

Work in the MW laboratory is funded by the BBSRC (grant BB/M019411/1) and the EU (Marie Curie Educational Training Network "INTEGRATE"). AC is supported by the Cambridge Trusts. EM is funded by a studentship from the MRC. SB is supported by a Hershel Smith studentship. E-FU is a clinical research fellow funded by the CF Trust (UK), Papworth Hospital NHS Trust and the Wellcome Trust. YA is supported by a scholarship from the Yosef Jameel Foundation. YB is an EPSRC-funded PhD student. Work in the laboratory of AF is supported by the Wellcome Trust. Work in the DRS laboratory is supported by the EPSRC. 
Figure Legends.

533 Figure 1. Virulence factors and their associated secretion systems in $\boldsymbol{P}$. aeruginosa. The genes encoding type I secretion systems ("T1" in the figure) are generally tightly-associated with their respective substrates (e.g., the aprDEF cluster encodes the alkaline protease T1 secretion system, which is located immediately adjacent to the genes encoding its secreted substrates, AprX and AprA). In contrast, the passenger proteins secreted through the type II secretion systems (T2) are distributed around the genome and carry cleavable $\mathrm{N}$-terminal signal sequences or Tat signals. Two $\mathrm{T} 2$ systems have been described in P. aeruginosa; the Xcp system [48] (which transports most of the proteins shown in the figure) and the Hxc system [49] which exports the alkaline phosphatase, LapA. The T3 and T6 systems are "injectisomes" which export proteins directly from the bacterial cytosol into the recipient cell [50], which may be either a host cell or another bacterium. P. aeruginosa encodes one dedicated type III secretion system (T3SS) comprised of around 40 genes, and three T6SSs (HSI-I, HSIII and HSI-III) each comprising around 15-20 genes. However, the flagellar apparatus (also a virulence factor in its own right, but not shown for clarity in the figure) can arguably be defined as a T3SS too. Finally, $P$. aeruginosa also encodes two varieties of T5SS [51]; the T5a autotransporter secretes the cell surface-associated esterase EstA whereas the T5b "two-partner" systems secrete e.g., the protease LepA (which targets NF-KB) or the chaperone usher protein, CupB5. There are four other T5b systems known to be encoded in the $P$. aeruginosa genome. In addition, $P$. aeruginosa also secretes polysaccharides such as Psl [15] and Pel [52], which are thought to constitute the biofilm/pellicle matrix, as well as alginate (whose production is essentially pathognomic of CF).

Figure 2. Reciprocal regulation of virulence factors in laboratory cultures: the "lifestyle paradigm". In laboratory growth conditions, planktonic cultures of $P$. aeruginosa secrete large quantities of tissuedamaging exoproteins, primarily due to the operation of the T1 and T2 secretion systems (see Figure 1). Expression of the $T 1$ and $T 2$ secretion systems and of many of their substrates is controlled by quorum sensing (QS) [5, 6]. In contrast, expression of the T3 system is only minimally affected by QS. Instead, T3 secretion - which leads to the subversion of host cell function - thought to be primarily turned "on" as a consequence of RetS signalling. In the activated state, RetS forms inhibitory heterodimers with the sensor kinase, GacS. This leads to less GacA phosphorylation and consequently, less expression of the RsmA antagonistic RNA species, rsmZ and rsmY. RsmA activity therefore becomes unfettered, leading to increased T3 secretion and motility and a concomitant decrease in T6 secretion/ps/ gene expression [45]. This reciprocal regulation is further fine-tuned by cyclic di-GMP levels (low cyclic-di-GMP concentrations favour motility and T3 secretion). In contrast, when a biofilm forms, LadS signalling dominates. This leads to a decrease in free RsmA levels, and combined with high cyclic-di-GMP concentrations, promotes increased T6 secretion and ps/ gene expression. Oddly, QS has been posited as being important for biofilm formation too, yet in vitro-grown biofilms exhibit only low levels of T1/T2-dependent protein secretion [4]. It is not yet clear why QS should impact so differently on protein secretion during planktonic and biofilm growth.

Figure 3. Overview of the TnSeq method. A library of in vitro-generated Tn mutants is prepared and is used to inoculate a laboratory culture and an animal infection model. After the requisite amount of time, bacterial DNA is harvested from each sample and the DNA flanking the Tn insertions is PCR amplified. Bar-coded sequencing primers are then ligated to each PCR product and the mixture is sent for DNA sequencing (usually using an Illumina sequencer). The quantitative nature of Illumina sequencing means that the number of reads associated with each Tn insertion can be determined, allowing the representation of each Tn mutant in the output pool (cf. the input pool or control growth 
condition) to be established. In the figure, Tn insertions in "gene B" are strongly negatively-selected in the mouse infection model, so the read frequency of $\mathrm{Tn}$ insertions in that gene/condition are negligible. This indicates that gene $B$ is essential for in vivo infection.

Figure 4. Overview of the glyoxylate shunt pathway in $\mathbf{P}$. aeruginosa. In the glyoxylate shunt (pale orange arrows and metabolites) carbon skeletons are redirected away from the $\mathrm{CO}_{2}$ producing steps of the TCA cycle and retained for gluconeogenesis. The glyoxylate shunt requires two enzymes: isocitrate lyase (ICL) and malate synthase (GICB), and the net effect of their activity is to convert two molecules of acetyl-CoA into one molecule of malate (a gluconeogenic precursor). In the chronic and acute wound models, the acetyl-CoA is generated as a consequence of FaoAB-mediated fatty acid oxidation. Unlike E. coli, in P. aeruginosa there are two isocitrate dehydrogenase enzymes (ICD and IDH), only one of which (ICD) is likely to be inhibitable by AceK-mediated phosphorylation [53]. 
Future

Figure 1

609

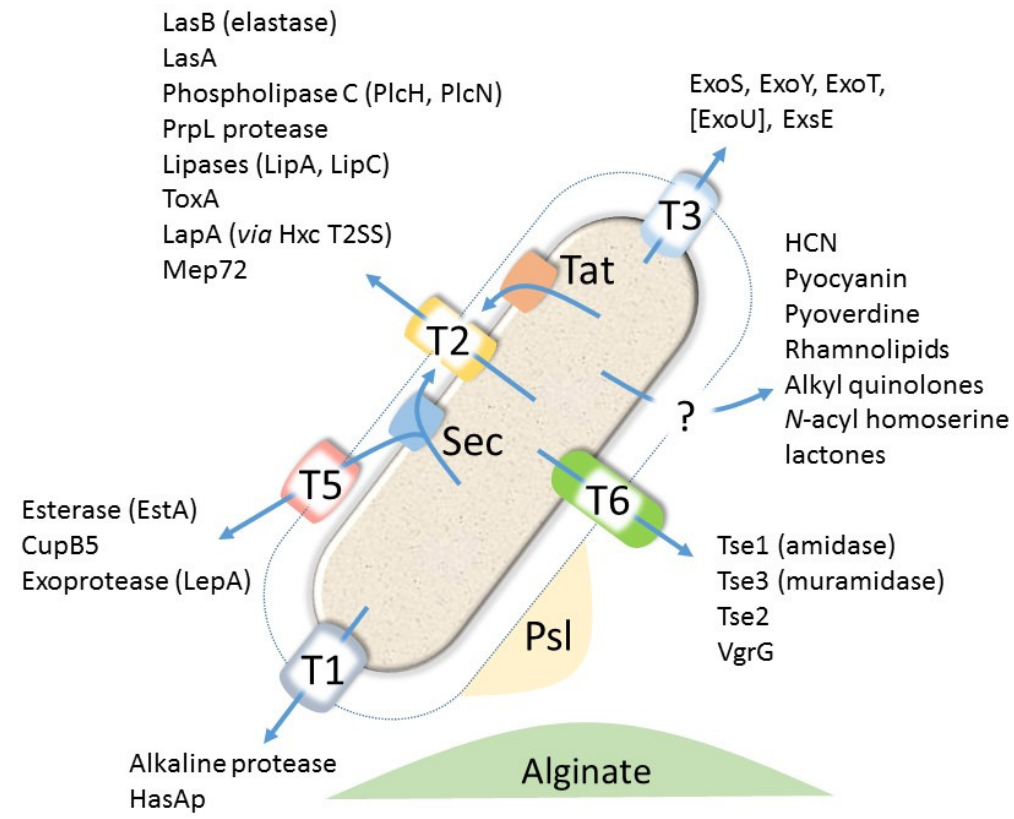

610 Figure 1

611

612

613

614

615

616

617 
Figure 2

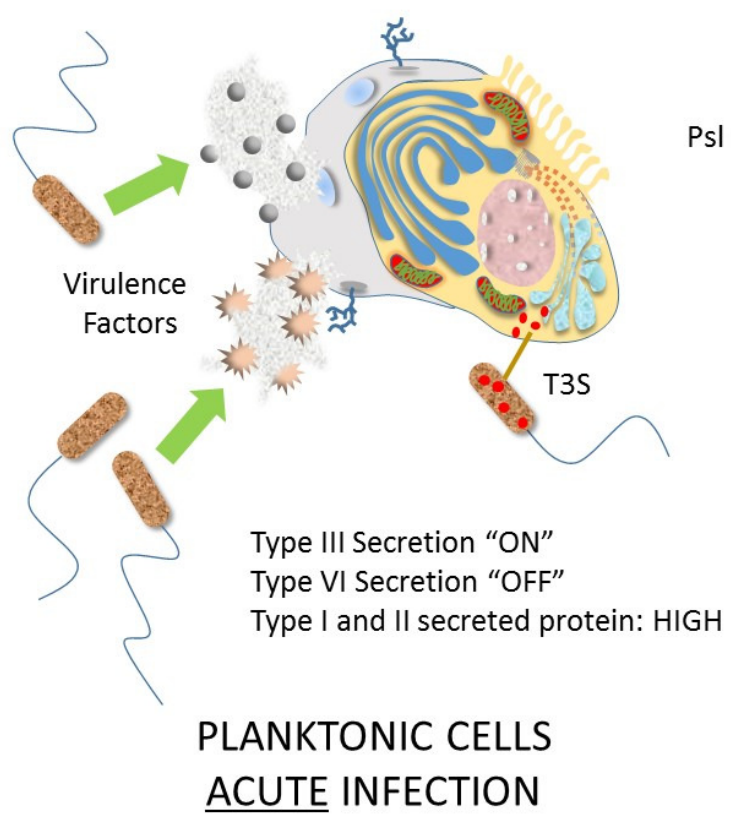

618

619

620

621

622

623

624

625

626

627

Figure 2

Type VI Secretion "ON" Type III Secretion "OFF" Type I and II secreted protein: LOW

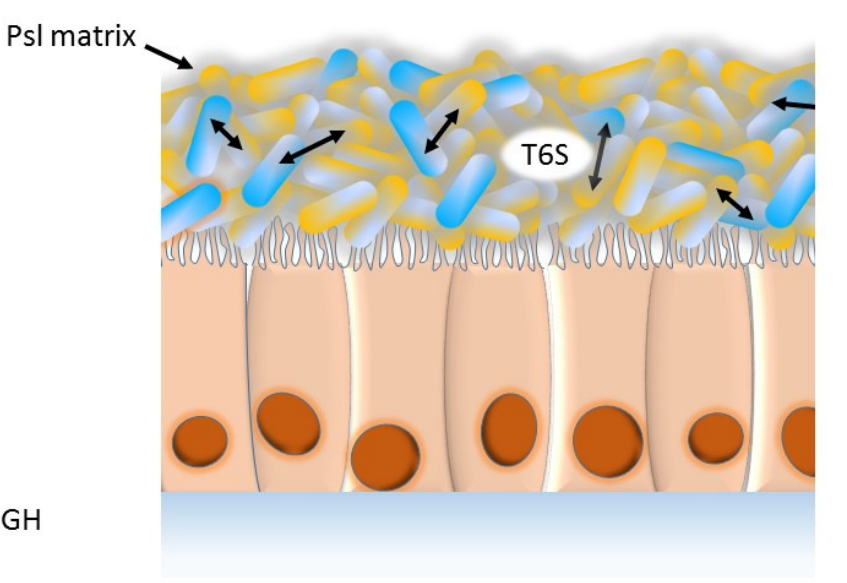

BIOFILM CELLS

CHRONIC INFECTION 
Figure 3

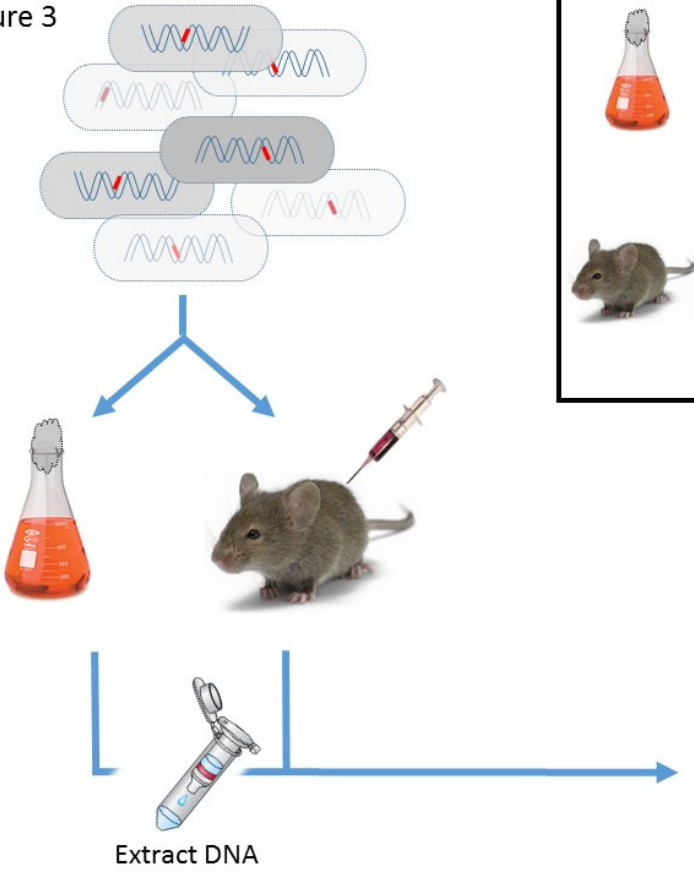

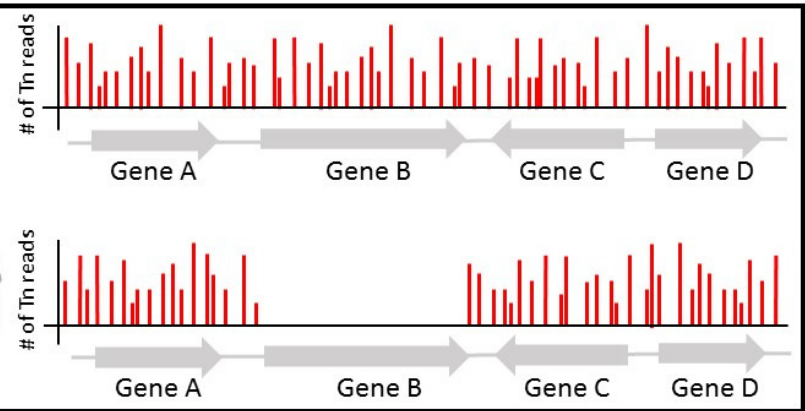

$\uparrow$

Quantitative Illumina Sequencing

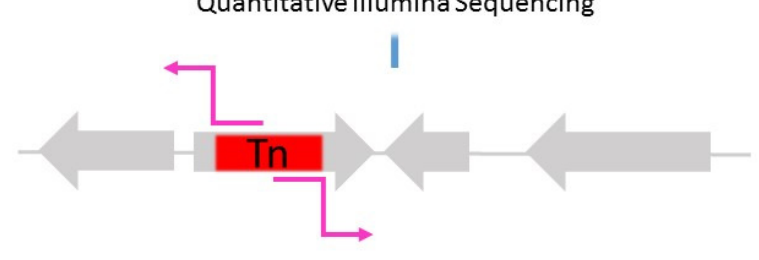

PCR amplify regions flanking $T n$ insertions in DNA extracted from each sample, ligate Illumina sequencing/bar coding adapters.

Figure 3

630 
Future

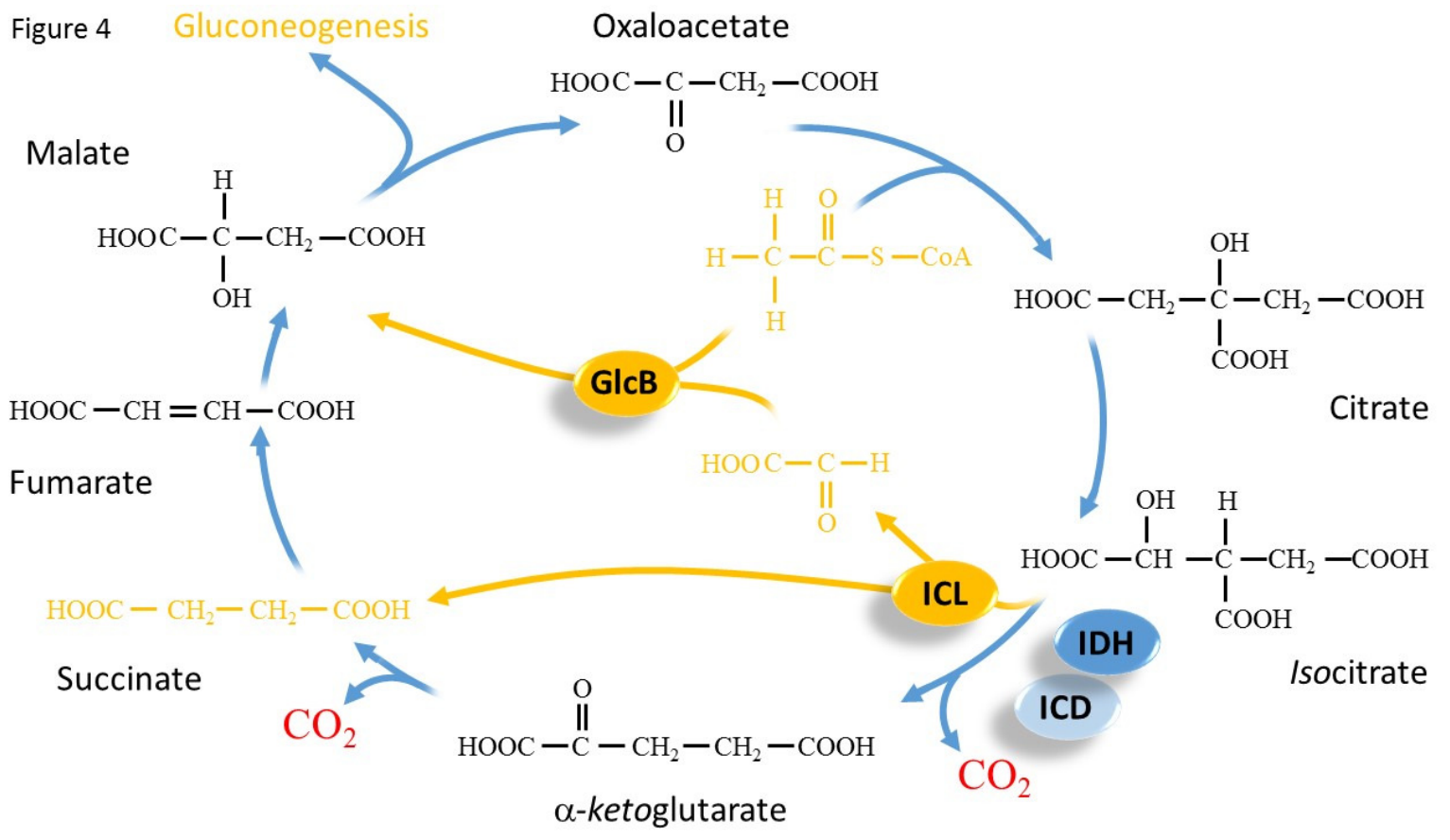

638

Figure 4 